\title{
Investigating R-Mode Signals for the VDE System
}

\author{
M. Wirsing, A. Dammann, R. Raulefs \\ Institute of Communications and Navigation \\ German Aerospace Center (DLR) \\ Oberpfaffenhofen, Germany \\ Email: \{Markus.Wirsing, Armin.Dammann, Ronald.Raulefs\}@DLR.de
}

\begin{abstract}
As GNSS signals are not always reliable and subject to jamming and spoofing, it is desired to have alternative means of maritime navigation. One approach for that is to equip communication systems on the shore with the option to transmit ranging signals (R-mode), which vessels can use to determine their position. In this paper, we show that by using the VDES system, which is currently in the process of standardization, it is possible to achieve significantly better ranging performance than by utilizing the Automatic Identification System (AIS) for this purpose.
\end{abstract}

\section{INTRODUCTION}

Current position information of a vessel is relevant for safety and navigation applications. The automatic identification system (AIS) utilizes the information by broadcasting it together with heading, current velocity and more detailed information about the vessel itself. The Global Navigation Satellite Systems (GNSSs) are the primary systems determining positions and its derivatives, such as velocity and heading. Recent events have shown that the satellite based systems are a single point of failure to determine the location. Therefore, it is desirable to have a secondary method of localization for monitoring the integrity of the GNSS and as well for navigation with reduced accuracy in case the satellite system fails. Such failure could be a solar storm which primarily affects space based systems, but could also be a local event in case of intentional jamming or even spoofing.

Therefore, the International Association of Lighthouse Authorities (IALA) discusses the need of a contingency or even backup system of the satellite systems. A backup system requires to fulfill similar performance requirements as the primary system, such as GPS. A contingency system has lower performance requirements, especially the holdover time until the clock of the transmitters causes significant errors in determining the position. However, both system requirements shall assure a safe travel of a vessel back to the harbour in case of a significant GNSS failure. The proposal is called $R$ Mode where $R$ stands for ranging. R-Mode intends to utilize the shore based communication infrastructure, like Automatic Identification System (AIS) base stations [1] with the existing housing infrastructure. The accuracy of ranges between the base station on land and the vessel at sea, measured by means of the received radio signal, depends on the utilized bandwidth and of the received signal power versus the power of other noise sources. The AIS system offers $25 \mathrm{kHz}$ of bandwidth and uses a GMSK modulated signal. Currently,
IALA defines a future version of AIS that is called VHF data exchange system (VDES) and comprises of the existing AIS, and an additional application message service (ASM) and the VHF data exchange (VDE). VDE has a terrestrial and a satellite component. The terrestrial component offers in the up- and downlink $100 \mathrm{kHz}$ each. Investigations have shown performance results from AIS and VDE. The authors in [2] investigate the AIS system and its existing infrastructure and how to use for ranging and positioning. A key aspect that was stressed is the impact of the geometric dilution of precision and limits on the coastal areas. Further, it was concluded that because of cost issues that a precise clock on the vessel seems unfeasible. An additional secondary factor was applied which increased the performance by an order of magnitude. However, it remains open how with the limited bandwidth of AIS the performance of about $10 \mathrm{~m}$ could be achieved. The concept of using AIS as a ranging source was further proposed and addressed in [4]. The concept described the application of RMode in the Baltic sea which comes in relevant areas with a favourable geometric dilution of precision (GDOP). The concept is expanded further to the MF frequency band to increase the coverage of individual transmitter sites. Therefore, a joint R-Mode system concept utilizing AIS and MF was proposed. The MF-R-Mode has significant advantages by using only limited infrastructure as the propagation conditions during day time can reach several hundred $\mathrm{km}$. However, during the night the skywave effect causes severe multipath errors which prohibit a night-time use of MF-R-Mode. In [5] the authors derived for AIS, ASM and VDE the CramerRao bounds and focused on the different bandwidths of both systems. They also considered five successive time slots in AIS and three successive time slots for ASM to improve the ranging performance and to compete with the terrestrial 100 $\mathrm{kHz}$ VDE system. The authors applied the ITU-R P.15465 channel model with distinct antenna heights to provide realistic assessments about the expected range. The authors concluded that the focus should be on VDE because of the superior performance results. In this paper we follow the path to apply VDE for ranging, and in addition shape signal to improve the performance and utilize the existing bandwidth further. We This follows the concept of [6] that optimized the Cramér-Rao bound to improve the ranging performance. Further, we applied the newly developed ranging sequence and performed measurements on the Lake Ammer in Bavaria. Sec. II introduces in detail the VDE signal structure. Sec. III 
discussed several ranging sequences. Sec. IV] outlined the theoretical analysis and is followed by a detailed description and results of the different measured sequences in Sec. V

\section{VDE SYSTEM OVERVIEW}

The VHF Data Exchange System (VDES) comprises of three different systems: AIS, ASM and VDE link [3]. The VDE link is currently being standardized, and offers new opportunities compared to the existing AIS. The VDE link differs from AIS in a few significant aspects: While AIS uses $25 \mathrm{kHz}$ of bandwidth, and has two channels available for use by either a base station or a vessel, VDE link is designed for two frequency blocks of $100 \mathrm{kHz}$ each; with one being dedicated to the uplink, and the other being reserved for downlink communications.

Within these $100 \mathrm{kHz}$ blocks, the VDES standard offers multiple transmission modes, either utilizing all of the available bandwidth for a single channel, or dividing it up into four channels of $25 \mathrm{kHz}$ each.

Another difference is the utilized modulation scheme. Unlike AIS, VDE does not rely on continuous phase modulation, and instead utilizes a linear modulation scheme. As linear modulation does not have a constant amplitude, thus the Peak to Average Power Ratio (PAPR) is a concern. In order to keep the PAPR levels acceptable, the $\pi / 4$-QPSK symbol mapping is used.

\section{Possible RANGING SIGNALS}

In order to determine the range between the vessel and a land based VDE base station, the base station needs to transmit a known signal whose time of arrival can be accurately estimated at the receiver. As the VDE system is intended to be used, these signals should conform to the VDES physical layer, so they can be used without disrupting equipment which is unprepared for their existence.

In order to determine the performance of different signals, we first consider an estimation theoretical approach. The Cramér-Rao bound (CRB) [7] is a bound that determines the minimum variance of an estimated variable. For the estimation of time of arrival, it states:

$$
\operatorname{var}(\hat{\tau}) \geq \mathrm{CRB}=\frac{1}{\frac{E}{N_{0} / 2} \omega^{2}},
$$

with $\omega$ being the effective bandwidth defined by

$$
\omega^{2}=\frac{\int_{-\infty}^{\infty}(2 \pi f)^{2}|S(f)|^{2} \mathrm{~d} f}{\int_{-\infty}^{\infty}|S(f)|^{2} \mathrm{~d} f} .
$$

The ranging performance therefore depends on the signal bandwidth.

As the VDES standard allows different bandwidths to be used, a few options are available.

- Using a single $25 \mathrm{kHz}$ channel: This option would make use of the possibility in the VDES standard to utilize a lower bandwidth with 4 channels instead of one wider channel. This way, the three other channels would still be available for communication while a ranging signal is

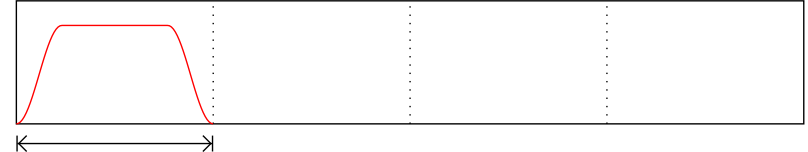

$25 \mathrm{kHz}$

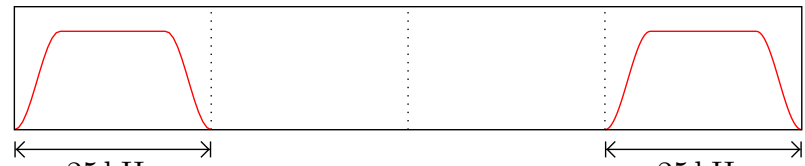

$25 \mathrm{kHz}$

$25 \mathrm{kHz}$

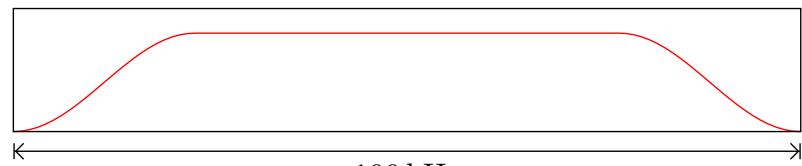

$100 \mathrm{kHz}$

Fig. 1. Different options for utilising the available bandwidth. Schematically showing the power spectral density of average VDE link signals.

being transmitted. It also serves as a useful reference, as it would occupy the same amount of frequency spectrum as an AIS signal.

- Using two $25 \mathrm{kHz}$ channels coherently: Using the two outermost $25 \mathrm{kHz}$ channels available in VDE, allows to achieve a high effective bandwidth, while possibly still leaving two channels available for communication purposes. Conformance to the VDE standard however needs to be determined.

- Using $100 \mathrm{kHz}$ : In this case, a signal is broadcasted using the full available bandwidth of the VDE system. Transmitting a data sequence which, under the constraints of the used $\frac{\pi}{4}$-QPSK, maximises the effective bandwidth, allows this option to reach good ranging performance.

The different options are visualised in Fig. 1.

\section{THEORETICAL CONSIDERATIONS}

In order to evaluate the possible performance of the different options, lower bounds on the estimation error for time of arrival estimation are used. Specifically, the Cramér-Rao bound and the Ziv-Zakai bound [8] are evaluated for the different signals. For a fair comparison between the different options, it is assumed that all of the considered signals have the same total energy. The results of the Ziv-Zakai bound are shown in Fig. 22 The Ziv-Zakai bound converges to the Cramér-Rao bound four high signal energies, but gives results closer to reality at low signal energies.

Already the use of a single VDE channel results in a significant improvement in ranging performance compared to AIS. This is due to the energy of the AIS signal being mostly located close to the center of the spectrum, thus resulting in a lower effective bandwidth. Utilizing two $25 \mathrm{kHz}$ channels results in further significant improvements over using only one channel, providing about six times better results than a signal with the same total energy but only $25 \mathrm{kHz}$ of bandwidth. Using the entire $100 \mathrm{kHz}$ bandwidth gives similar results when an optimized transmit sequence is chosen which puts most 


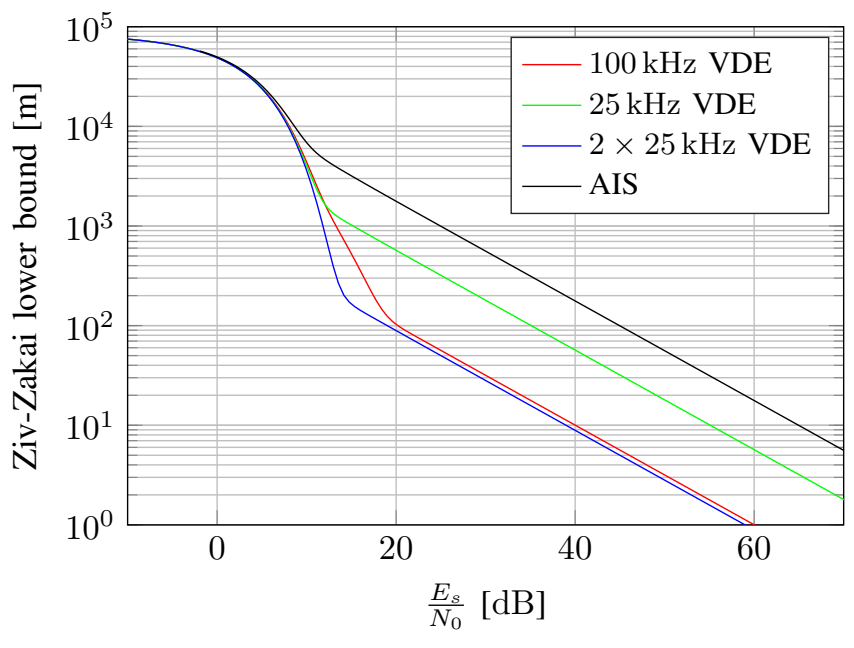

Fig. 2. Lower bounds on the time of arrival estimation error.

of the signal energy in the outer regions of the spectrum. Two $25 \mathrm{kHz}$ channels offer the option to use the remaining bandwidth for communication purposes. Implementation issues however might favour the use of the $100 \mathrm{kHz}$ channel.

Compared to AIS, the required signal energy for the same ranging performance can be reduced by about $24 \mathrm{~dB}$ by making use of two $25 \mathrm{kHz}$ VDE link channels. In case of constant transmitted signal energy, this would result in about 15 times more range, for the same ranging performance, when only free space coverage is considered.

\section{Measurements}

To validate the results obtained by considering estimator bounds, a measurement campaign was performed.

\section{A. Measurement Setup}

The measurement setup consisted of a transmitter on the land side and a receiver on a vessel on the lake. Both sides utilised Software Defined Radio (SDR) hardware to transmit or receive the different signals to be evaluated.

The location of the measurement was the Lake Ammer in Germany. As usage of motorboats is highly restricted on the lake, the measurement was performed on board of a commercial passenger ship. The antenna of RX side SDR, as well as the GNSS antenna can be seen in Fig. 5.

In addition to the same SDR hardware, as used on the receiver side, the TX side setup also included a power amplifier to allow transmission of the signal with a power of 1 Watt. A higher power was not approved by the regulatory agency. The transmitter antenna was mounted on top of a car, as is shown in Fig. 5, however the car remained stationary during the entire measurement.

\section{B. Measurement Methods}

In order to allow a fair comparison between the different ranging signals, they should be transmitted in close temporal proximity, such that the channel conditions are comparable. In

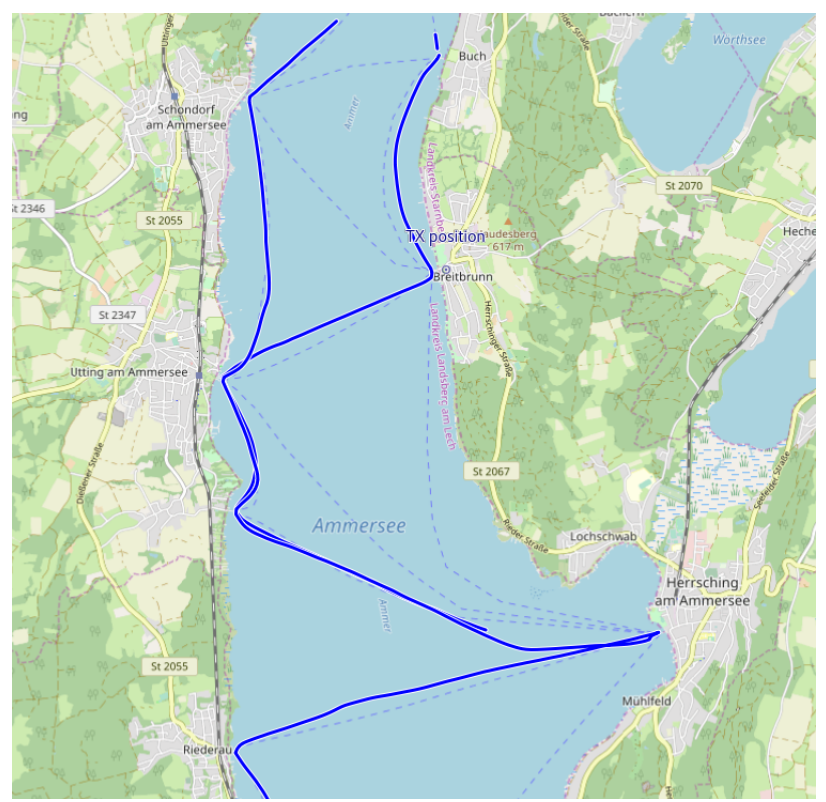

Fig. 3. Map of the Lake Ammer where the measurements were performed.

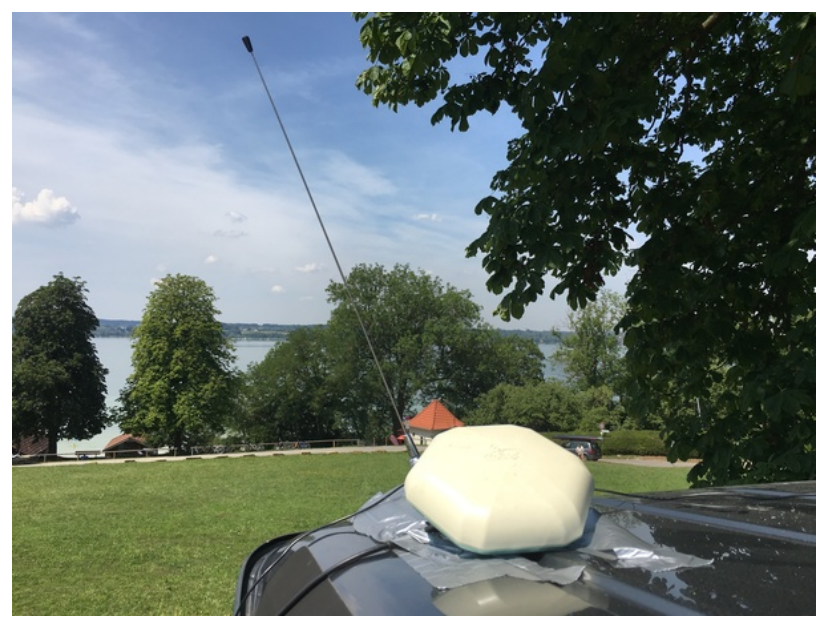

Fig. 4. Transmitter antenna next to the Lake

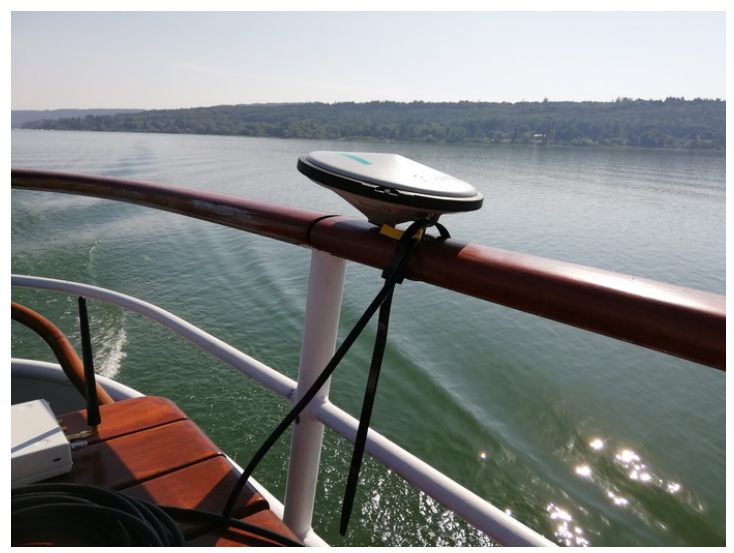

Fig. 5. Receiver antenna and GNSS antenna on the ship. 
our measurement, this was done by transmitting each signal of interest in succession with a guard interval in between.

Each block of transmitted signals was sent at the full second mark. Thus, the update rate for the measured range was $1 \mathrm{~Hz}$. A reference Pulse-Per-Second (PPS) for precise timing of the transmitted signal was provided by a GPS disciplined oscillator (GPSDO) module in the SDR hardware. The carrier frequency was also derived by a $10 \mathrm{MHz}$ signal provided by the GPSDO.

On the receiver side, the same type of SDR and GPSDO was utilized. This way, both sides had access to a common GNSS based time base. While the purpose of R-mode is to be independent of satellite based systems, the purpose of this measurement was only to evaluate the possible ranging signals. After the reception of each signal block, it was tagged with a time stamp and written to disk in the form of I/Q baseband samples.

As a reference for the true range between transmitter and receiver, both sides were equipped with GNSS receivers.

Evaluation of the recorded signals was done in a postprocessing step. As each signal was tagged with the time of reception, the data could be associated with the appropriate position as recorded by the GNSS equipment. The true range could then be calculated. To determine the estimated range from the measurements, first the appropriate time slot within the one second block of recorded samples was cut out. Then a coarse synchronisation was performed by determining the maximum of the cross correlation of the received signal with the transmitted signal:

$$
\hat{\tau}=T_{s} \cdot \underset{m}{\operatorname{argmax}}\left|\sum_{n=0}^{N-1} r(n) \cdot s^{*}(n-m)\right|^{2}
$$

Where $N$ is the number of samples within one timeslot, $r(n)$ denotes the received samples, and $s(n)$ is the transmitted signal.

As a sampling frequency $f_{s}$ of $612.5 \mathrm{kHz}$ was used, the result of this estimation is quantized to steps of $1.63 \mu \mathrm{s}$, which corresponds to a range of $489.5 \mathrm{~m}$. This is obviously unsatisfactory for the purpose of a ranging system. Therefore an additional fine estimation step is necessary.

As the fine synchronisation needs to determine the time of arrival of the signal with a better accuracy than the sampling time, it is necessary to interpolate between the recorded samples. This can be achieved by the means of a Discrete Fourier Transform (DFT). A cyclic shift in time domain corresponds to a multiplication with the complex exponential function in frequency domain. Thus a time shift can be achieved by multiplying the frequency domain representation of a sufficiently zero padded signal with an exponential function, and transforming the signal back to time domain. For the purpose of determining the maximum correlation, it is possible to omit the inverse DFT and do the correlation in frequency domain.

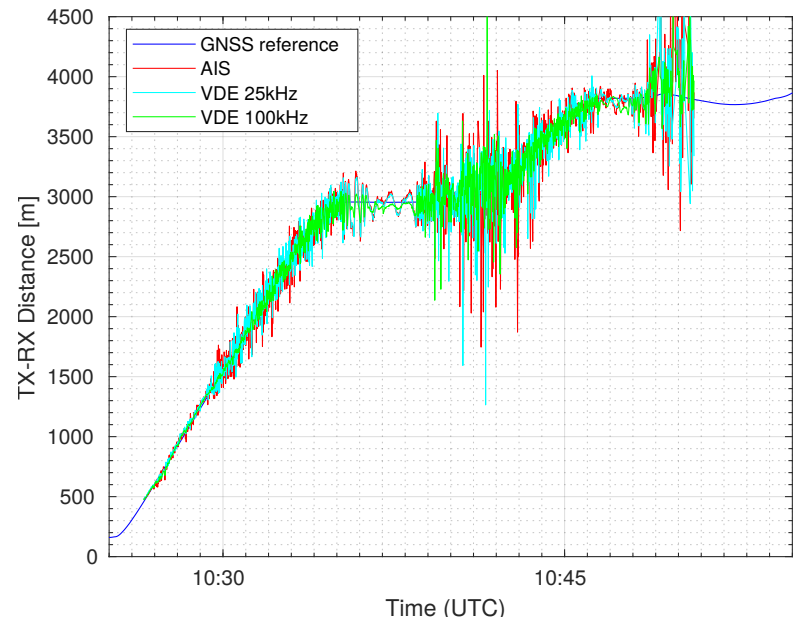

Fig. 6. Ranging performance plotted over time.

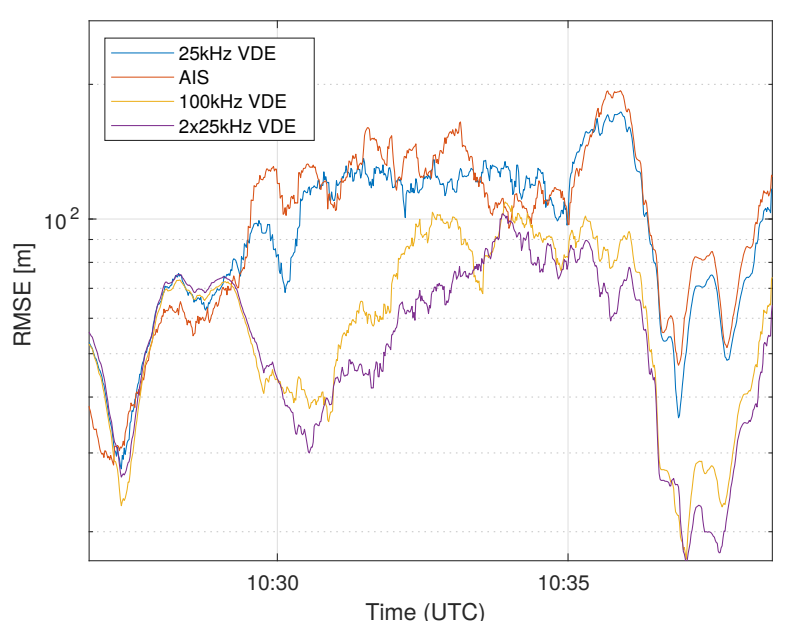

Fig. 7. RMSE in a sliding 30 second window

$$
\hat{\tau}=\underset{\tau}{\operatorname{argmax}}\left|\sum_{k=0}^{K-1} R(k) \cdot\left(S(k) \cdot \exp \left(-j 2 \pi \frac{\tau}{T_{s}} k\right)\right)^{*}\right|^{2}
$$

Where $\mathrm{R}(\mathrm{k})$ and $\mathrm{S}(\mathrm{k})$ are the DFT of the received and the transmitted signal. The maximum in Eq. (4) has to be found by numerical methods, as there are no analytical methods possible for the measured signal.

\section{Measurement Results}

As a first step, the data collected on the way from Breitbrunn to Utting was evaluated. The path of the ship can be seen on the map in Fig. 3 The result of the distance estimation plotted over time is shown in Fig. 6

In Fig. 6, it can already be seen, that the VDE signals provide a better estimate of the actual distance. However the measurement also exhibits some artifacts at about 10:37 UTC. It is not yet understood what causes this, however multipath 
effects could be an explanation. As the RX antenna, was not mounted at the highest point of the ship, parts of the ship itself could act as a reflector and cause the signal from a stronger path to arrive at the antenna.

As the graph in Fig. 6 does not allow a good comparison between the different signals, Fig. 7 shows the RMSE of the estimated ranges over a sliding window of 30 seconds. It can be seen that the signals with $100 \mathrm{kHz}$ of bandwidth perform noticeably better than AIS or VDE with $25 \mathrm{kHz}$.

\section{SUMMARY}

For the purpose of measuring ranges and calculating a position based on these ranges, significant improvements can be expected from the upcoming VDE system compared to the existing AIS. Theoretical considerations based on estimation theory and bounds on estimator errors suggest that using either a $100 \mathrm{kHz}$ channel or two $25 \mathrm{kHz}$ channels coherently provide the best results. The measurements that were performed, support this theory. However, further analysis of the measurement data is required, in particular taking into account signal propagation effects.

\section{REFERENCES}

[1] Gregory Johnson, Peter Swaszek, 2014, "Feasibility Study of R-Mode using AIS Transmissions", https://www.iala-aism.org/content/uploads/ 2016/08/accseas_r_mode_feasibility_study_ais_transmissions_part_2. pdf

[2] Qing Hu, Yi Jiang, Jingbo Zhang, Xiaowen Sun and Shufang Zhang, 2015 "Development of an Automatic Identification System Autonomous Positioning System", doi:10.3390/s151128574

[3] IALA Guideline G1139, "The Technical Specification of VDES", https: //www.iala-aism.org/product/g1139-technical-specification-vdes/

[4] Gewies, Stefan und Dammann, Armin und Ziebold, Ralf und Bäckstedt, Jesper und Bronk, Krzysztof und Wereszko, Błażej und Rieck, Carsten und Gustafson, Per und Eliassen, Cato Giil und Hoppe, Michael und Tycholiz, Wojciech (2018) R-Mode Testbed in the Baltic Sea. In: 19th IALA Conference 2018. 19th IALA Conference 2018, Incheon, SouthKorea, https://elib.dlr.de/120702/

[5] Jan Šafár, Alan Grant, Paul Williams and Nick Ward, "Performance Bounds for VDES R-mode", THE JOURNAL OF NAVIGATION, doi:10.1017/S0373463319000559

[6] Armin Dammann, Thomas Jost, Ronald Raulefs, Michael Walter and Siwei Zhang, "Optimizing waveforms for positioning in 5G", https:// elib.dlr.de/106279/1/SPAWC2016_PostPrint.pdf

[7] S. M. Kay, Fundamentals of Statistical Signal Processing - Estimation Theory. Prentice Hall, 1993.

[8] C. Musso and J.-P. Ovarlez, "Improvement of the Ziv-Zakai lower bound for time delay estimation," in 15th European Signal Processing Conference (EUSIPCO 2007), Poznan, Poland, Sep. 2007, pp. 960-964. 